Research Paper

\title{
Development of an Anti-Adhesive Membrane for Use in Video-Assisted Thoracic Surgery
}

\author{
Akiko Uemura ${ }^{1}$, Toshiharu Fukayama2, Takashi Tanaka ${ }^{1}$, Yasuko Hasegawa-Baba ${ }^{3}$, Makoto Shibutani ${ }^{3}$, \\ Ryou Tanaka ${ }^{1 凶}$ \\ 1. Tokyo University of Agriculture and Technology Animal Medical Center, 3-5-8 Saiwaicho, Fuchu-shi, Tokyo 183-8509, Japan \\ 2. National Cerebral and Cardiovascular Center, 5-7-1 Fujishiro-dai, Suita, Osaka 565-8565, Japan \\ 3. Laboratory of Veterinary Pathology, Tokyo University of Agriculture and Technology, 3-5-8 Saiwai-cho, Fuchu-shi, Tokyo 183-8509, Japan \\ $\square$ Corresponding author: Ryou Tanaka, Department of Veterinary Surgery, Tokyo University of Agriculture and Technology Animal Medical Center, Tokyo \\ 183-8509, Japan. Tel: +81-42-3675904; Fax: +81-42-3675904; E-mail: ryo@vet.ne.jp \\ (c) Ivyspring International Publisher. This is an open access article distributed under the terms of the Creative Commons Attribution (CC BY-NC) license \\ (https:// creativecommons.org/licenses/by-nc/4.0/). See http://ivyspring.com/terms for full terms and conditions.
}

Received: 2017.11.27; Accepted: 2018.04.12; Published: 2018.04.27

\begin{abstract}
Background: The need to prevent postoperative adhesions after surgery has been considered a significant challenge in thoracic surgery, especially with the advent of video-assisted thoracic surgery (VATS). While preventive materials for postoperative adhesions have been studied for many years, they are all still in the development phases.

Methods: In this animal study, an insoluble hyaluronic acid membrane was used in VATS for wedge resection to test its operability and to examine the body's response to the membrane. Ten beagles were divided into two groups, an experimental group and a negative control group. In the experimental group, an insoluble hyaluronic acid membrane containing glycerol was used as the test membrane $\left(10 \times 10 \times 0.1 \mathrm{~cm}^{3}\right)$. The test membrane was implanted in the left thoracic cavity of the animal under VATS following wedge resection. The animals were observed for two weeks and then euthanized for examination.

Results: Macroscopically, the median adhesion score was lower in the experimental group (0) than in the control group (2.5). On histopathological examination, the test membrane elicited only a minor inflammatory response and foreign body reaction.

Conclusion: The test membrane showed satisfactory operability and appears to be a practical material to prevent postoperative adhesions after thoracic surgery in VATS.
\end{abstract}

Key words: Preventing adhesion, VATS, thoracic surgery, insoluble hyaluronic acid membrane

\section{Introduction}

Postoperative adhesions occur at a high rate after surgery, and their adverse effects are widely recognized as peritoneal adhesions after abdominal surgery, which are known to cause organ disorders such as abdominal pain, ileus, and infertility [1, 2]. Moreover, such postoperative adhesions after abdominal surgery are known to occur after endoscopic surgery, as well as after laparotomy [3]. While postoperative adhesions also occur at a high rate after thoracic surgery, their association with organ disorders has rarely been reported [4]. Nonetheless, postoperative adhesions after thoracic surgery can cause major adverse effects in cases requiring repeated thoracic surgery [5-9].

In addition, problems with postoperative adhesions are also described in video-assisted thoracic surgery (VATS) [10-12]. Since the surgical manoeuvers available in VATS are restricted, the presence of adhesions is predicted to be a greater problem, because the surgical field of view is limited compared with thoracotomy. From the standpoint of VATS development in the future, the prevention of postoperative adhesions is the challenge. In prevention of adhesions after abdominal surgery, a 
film consisting primarily of a cellulose derivative (carboxymethyl-cellulose) was developed and subsequently commercialized. This has been shown to be effective in reducing postoperative adhesions after abdominal surgery $[13,14]$ and also in prevention of adhesions after thoracic surgeries in pediatric cardiac surgery [15], as well as in rat mediastinoscopy [10]. There are several methods by which carboxymethyl-cellulose membranes are used in laparoscopy [16-18], and this method has shown effectiveness in laparoscopy $[19,20]$. On the other hand, an anti-adhesion film for use in thoracic surgery is not commercially available [21-24].

An insoluble hyaluronic acid membrane containing glycerol has been developed that shows greater effects in preventing postoperative adhesions after thoracic surgery than the above-mentioned carboxymethyl-cellulose membrane [25]. In a previous study in which thoracotomy was performed in dogs with the use of a novel membrane that uses surface water induction technology to prevent adhesions (insoluble hyaluronic acid membrane), we also showed that this membrane is effective in preventing postoperative adhesions after thoracotomy [26].

Based on the above, it is predicted that materials that are highly effective in preventing postoperative adhesions after thoracic surgery and can be used in VATS with a small incision of about 3-6 cm [27] will become essential in cardiac and respiratory surgeries. In a previous study, when a large incision was made in situations such as thoracotomy, we were able to cover the whole target site by inserting the membrane into the thoracic cavity after gently folding it in half. However, because the membrane was not strong enough to withstand damage caused by solid instruments such as tweezers and forceps, their use in VATS surgery should be evaluated.

This study examined the operability, safety, and efficacy of an anti-adhesive insoluble hyaluronic acid membrane in VATS.

\section{Materials and Methods}

This study was approved by the Institutional Animal Care and Use Committee of Tokyo University of Agriculture and Technology (Permit number 27-36). All treatments involving experimental animals were conducted in accordance with the Animal Experiments Subcommittee of Tokyo University of Agriculture and Technology and the Guide for the Care and Use of Laboratory Animals Eighth Edition (Committee for the Update of the Guide for the Care and Use of Laboratory Animals; National Research Council).

\section{Test membrane implantation}

Ten male TOYO beagles (9.8-10.5 kg) were purchased from Kitayama Labs Co. Ltd. (Nagano, Japan). The experiment consisted of two groups: the experimental group and the control group ( $\mathrm{n}=5$ each). Animals were given cefovecin sodium $(8 \mathrm{mg} / \mathrm{kg}$, sc; Convenia ${ }^{\circledR}$, Zoetis Japan Inc., Tokyo, Japan) to prevent infection and buprenorphine $(0.02 \mathrm{mg} / \mathrm{kg}$, sc; Buprenorphine for injection $0.2 \mathrm{mg}$, Nissin Pharmaceutical Co., Ltd., Tokyo, Japan) for analgesia. Subsequently, animals were pre-treated with atropine sulphate, butorphanol tartrate $(0.2 \mathrm{mg} / \mathrm{kg}$, iv; Vetorphale $^{\circledR}$, Meiji Seika Pharma Co., Ltd., Tokyo, Japan), and midazolam (0.2 mg/kg, iv; Midazolam injection [SANDOZ], Sandoz K.K., Tokyo, Japan), followed by general anesthesia induction with propofol (6 mg/kg, iv; "Mylan," Mylan Inc., Tokyo, Japan). Following tracheal intubation, anesthesia was maintained with isoflurane inhalation (1-2\%, Isoflurane for animals, Intervet K.K., Tokyo, Japan). Respiratory management was performed with manual bag-mask ventilation and intermittent positive pressure breathing through an artificial anesthesia device.

An insoluble hyaluronic acid membrane containing glycerol was used as the test membrane (10 $\mathrm{cm} \times 10 \mathrm{~cm} \times 0.1 \mathrm{~cm})$. The test membrane was implanted in the left thoracic cavity of the animal under VATS. A 12-mm-diameter port was created at the tenth intercostal space on the left side with a trocar, and a 35-mm-diameter small incision for operation was subsequently created at the fifth intercostal space on the left side under video camera monitoring. A wound protector (for 35-mm-diameter incisions) (Wrap Protector FF0707, Hakko Co., Ltd., Nagano, Japan) was inserted at the small incision for operation. Intercostal nerve block was performed in advance with bupivacaine (Marcaine injection 0.5\%, AstraZeneca plc, Osaka, Japan) for port and small incision sites. An automatic suture device (Endo GIA, $45 \mathrm{~mm}$, Covidien Japan Inc., Tokyo, Japan) was inserted from the port at the tenth intercostal space. Grasping forceps were then inserted from the small incision to hold the lung parenchyma, and the automatic suture device was used for stapling and dissection. Then, dissected lung tissue was removed from the small incision. In the experimental group, after the adhesion-preventing membrane was inserted from the small incision and placed between the visceral pleura and parietal pleura, and placed the center of the test membrane just under the small incision. A drain tube (Phycon tube SH No. 3: $2.5 \mathrm{~mm}$ inner diameter, $4.0 \mathrm{~mm}$ outer diameter, Fuji Systems Corporation, Tokyo, Japan) was inserted. After gradually re-expanding the lung lobes, the trocar was 
removed. The wound was closed using $2 / 0$ synthetic absorbable suture (Biosyn, Covidien Japan Inc.) using a conventional method. For the control group, a similar procedure was used without inserting the adhesion-preventing membrane, and the wound was subsequently closed.

Any abnormalities such as pneumothorax and pleural effusion were checked on the day after surgery. Pleural effusions were removed, if present, once a day, and their volumes were recorded. Chest drains were removed when pleural effusions were no longer observed.

At postoperative week 2, animals were anaesthetized similarly to the operation for membrane insertion and then euthanized with an overdose of potassium chloride solution under deep general anesthesia. Subsequently, blood was removed, and the chest was re-opened with median sternotomy.

\section{Observation and test methods}

The day of implantation was specified as day 1 of observation. At the time of sacrifice when the chest was re-opened, adhesions, if present, were dissected macroscopically using Kelly forceps, Metzenbaum scissors, and cotton swabs, and the strength of adhesions was evaluated and scored based on the degree of bluntness or sharpness of the dissection process $(0=$ no need to dissect; $1=$ film-like adhesion, can be dissected easily; $2=$ mild adhesion, can be dissected; $3=$ moderate adhesion, difficult to dissect; $4=$ strong adhesion, impossible to dissect), using the same scoring systems as in a previous report [26]. The macroscopic findings of adhesions after thoracotomy were compared statistically by comparing the adhesion scores of the Experimental group and the Control group using the Mann-Whitney U test.

For histological examination, parietal pleura and lung samples were collected near the test membrane insertion site. Samples were taken from two parts. The one was from the parietal pleura adjacent to the suture site of the small incision of the fifth intercostal space. The other one was from the visceral pleura adjacent to lung resection site in the cranial lobe of the left lung. Removed pleural and lung tissues were fixed in $10 \%$ neutral buffered formalin solution for one week at room temperature. After fixation, intercostal tissues were cut perpendicularly from the parietal to the visceral direction to create tissue slice samples that were embedded in paraffin blocks. After sectioning, samples were stained with hematoxylin and eosin (HE). Under an optical microscope, histopathological lesions were categorized according to the criteria for histopathological classification described below, and images of a representative view for each finding were taken. To compare the effects of the test membrane in preventing adhesions, the adhesion site and dorsal aspect of the lungs (including visceral pleura) were histopathologically evaluated in terms of tissue adhesion, fibrosis, mesothelial cell hypertrophy, cuboidal epithelialization of type II alveolar epithelial cells, and mononuclear cell infiltration in animals with adhesions between the lung and chest wall and in animals with interlobular adhesions. In animals without adhesions, the dorsal aspect of the lungs (including visceral pleura) was similarly evaluated.

The criteria for histopathological classification were the following: (adhesion: -, absent; + , present), (fibrosis in pleura: +, localized; ++, diffuse), (mesothelial cell hypertrophy in pleura: -, absent; +, mild), (alveolar epithelial cell cuboidal epithelialization in alveoli: -, absent; +, localized; ++, diffuse), (mononuclear cell infiltration in alveoli: -, absent; +, localized), and (mononuclear cell infiltration in interstitium: -, absent; +, localized; ++, diffuse).

\section{Results}

\section{Insertion of an adhesion prevention membrane}

After immersion in saline, the membrane became rapidly and sufficiently pliable, and it was not cracked by normal handling. In one animal (E4), the membrane was torn into multiple pieces while delivering it from the small $\sim 3.5-\mathrm{cm}$ incision for left-sided VATS in the thoracic cavity, making it difficult to completely cover the target site. Membrane insertion in all other animals in the experimental group was achieved successfully (Fig 1). Moreover, there were no differences in operability with wet gloves or with a wet wound protector placed at the small incision site. The membrane did not hinder the chest closing procedure.

\section{Macroscopic findings after thoracotomy}

In the experimental group, adhesions were observed between the chest wall and lungs in $2 / 5$ animals, and blunt dissection of the adhesions was difficult to achieve in one animal (E4) (adhesion scores: 3 for E4, 0 for E5, 2 for E6, 0 for E7, and 0 for C10). Pulmonary interlobular adhesions were observed in 2/5 animals, but blunt dissection could be achieved in all adhesions (adhesion scores: 0 for E4, 0 for E5, 0 for E6, 2 for E7, and 2 for E10). The median adhesion score was 0 . In the control group, adhesions were observed between the chest wall and lungs in $3 / 5$ animals, and blunt dissection of the adhesions was difficult to achieve in one animal (C2) (adhesion scores: 0 for $\mathrm{C} 1,3$ for $\mathrm{C} 2,0$ for $\mathrm{C} 3,1$ for $\mathrm{C} 8$, and 1 for 
C9). Interlobular adhesions were observed in all 5/5 animals, and blunt dissection was difficult to achieve in 4 animals (adhesion scores: 3 for C1, 3 for C2, 2 for C3, 4 for C8, and 3 for C9). The median adhesion score was 2.5. (Table 1) The adhesion score of the Experimental group was significantly lower $(\mathrm{P}<0.01$, Mann-Whitney $U$ test). In both the implant group and the control group, adhesions were completely absent at the VATS insertion port (tenth dorsal intercostal space).

With regard to the pleural effusions, $4 / 5$ animals in the experimental group (excluding E4) showed pale yellow, viscous pleural effusions $(90-110 \mathrm{~mL} / \mathrm{dog}$ ) (Fig 2). Most of the test membranes showed a mucoid-like appearance, with some showing a mass of a few mm mixed in the pleural effusion.

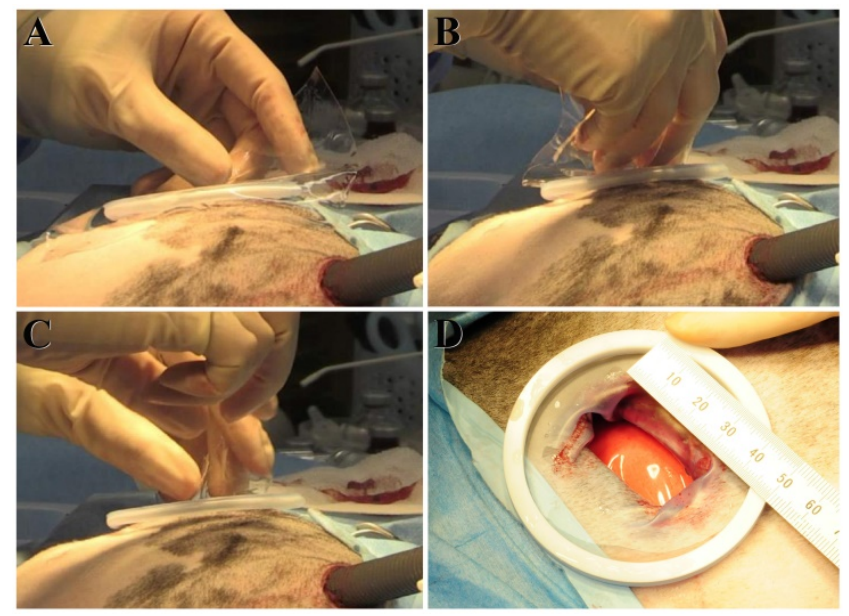

Fig 1. Membrane insertion procedure. In the experimental group, the adhesion-preventing membrane is inserted from the small incision and placed between the visceral pleura and parietal pleura. Membrane insertion in all animals except for E4 in the experimental group was achieved successfully in the order of panel A to panel D.

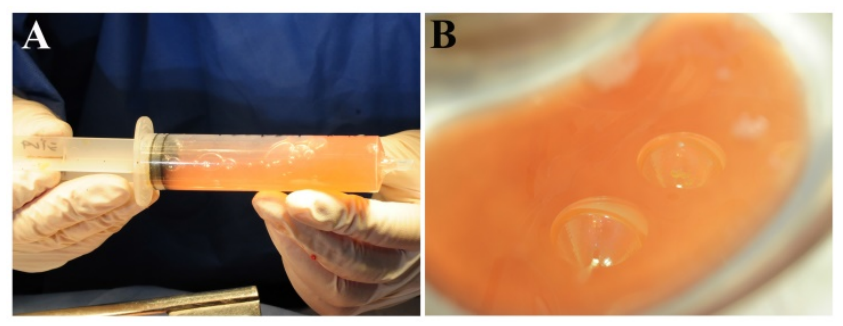

Fig 2. Pleural effusions observed at the re-thoracotomy. Four of five animals in the experimental group (excluding E4) show pale yellow, viscous pleural effusions $(90-110 \mathrm{~mL} / \mathrm{dog})$.

\section{Histopathological evaluation}

There were no obvious difference between the experimental group and the control group in the incidence or severity of adhesion of the lung and chest wall, pulmonary interlobular adhesion, pleural fibrosis (Fig 3A and B), pleural mesothelial cell hypertrophy (Fig 3C and D), alveolar epithelial cell cuboidal epithelialization (Fig 3E and F), alveolar mononuclear cell infiltration (Fig $3 \mathrm{E}$ and $\mathrm{F}$ ), and interstitial mononuclear cell infiltration (Table 2).

Table 1. Macroscopic findings of adhesions after thoracotomy

\begin{tabular}{lll}
\hline & Control group & Experimental group \\
\hline $\begin{array}{l}\text { No. of animals examined } \\
\text { Adhesion score }\end{array}$ & 5 & 5 \\
$\begin{array}{l}\text { Between the chest wall and lungs } \\
(1 / 2 / 3 / 4)\end{array}$ & $3(2 / 0 / 1 / 0)$ & $2(0 / 1 / 1 / 0)$ \\
$\begin{array}{l}\text { Pulmonary interlobular adhesions } \\
(1 / 2 / 3 / 4)\end{array}$ & $5(0 / 1 / 3 / 1)$ & $2(0 / 2 / 0 / 0)$ \\
Median adhesion score & 2.5 & 0.0
\end{tabular}

Adhesion scores: the degree of bluntness or sharpness of the dissection process $(0$, no need to dissect; 1 , film-like adhesion, can be dissected easily; 2 , mild adhesion, can be dissected; 3 , moderate adhesion, difficult to dissect; 4 : strong adhesion, impossible to dissect).

Table 2. Incidence of histopathological changes in the left lung and left chest wall

\begin{tabular}{lll}
\hline & Control group & Experimental group \\
\hline $\begin{array}{l}\text { No. of animals examined } \\
\text { Adhesions }\end{array}$ & 5 & 5 \\
$\quad$ Lung and chest wall $(+)$ & $1(1)$ & $2(2)$ \\
$\quad$ Pulmonary interlobular $(+)$ & $3(3)$ & $2(2)$ \\
$\begin{array}{l}\text { Pleura } \\
\quad \text { Fibrosis }(+/++) \\
\quad \text { Mesothelial cell hypertrophy }(+)\end{array}$ & $5(2 / 3)$ & $5(0 / 5)$ \\
$\begin{array}{l}\text { Alveoli } \\
\quad \text { Epithelial cell cuboidal } \\
\text { epithelialization }(+/++)\end{array}$ & $3(3 / 0)$ & $5(5)$ \\
$\quad$ Mononuclear cell infiltration $(+)$ & $3(3)$ & $5(4 / 1)$ \\
$\begin{array}{l}\text { Interstitium } \\
\quad \text { Mononuclear cell infiltration }(+/++)\end{array}$ & $4(4 / 0)$ & $1(1)$ \\
\hline
\end{tabular}

Criteria for histopathological classification: Adhesions: + , present; Fibrosis in the pleura: + , localised; ++ , diffuse; Mesothelial cell hypertrophy in the pleura: + , mild; Alveolar epithelial cell cuboidal epithelialization: + , localised; ++, diffuse; Alveolar mononuclear cell infiltration: + , localised; Interstitial mononuclear cell infiltration: + , localised; ++ , diffuse.

\section{Discussion}

One of the major differences between thoracotomy and VATS is the size of the incisions associated with the surgery [27]. The insoluble hyaluronic acid membrane can be cracked and torn into multiple pieces if it is completely folded. In fact, in one animal of the experimental group (E4), the membrane tore into several pieces during its delivery from the small incision to the thoracic cavity, and complete coverage of the target site could not be achieved. However, it was possible to insert the test membrane by grasping the four corners of the test membrane. With this procedure, the membrane forms a drawstring pouch and can be dropped into the thoracic cavity by pushing the center of the membrane into the thoracic cavity, without folding it from the small incision. Although it is necessary to have some experience to insert the membrane from a small incision, the technique does not require skillful 
technique. Moreover, this procedure can be achieved with both wet and dry surgical instruments and gloves. The membrane showed adhesion-preventing effects simply by placing the membrane without attachment to wrap the target site. From this point of view, this membrane was superior to the carboxymethyl-cellulose membrane for laparoscopy use. The size of the small incision created in the present study $(\sim 3.5 \mathrm{~cm})$ was similar to the typical size of incision that is created to remove pulmonary lobes in clinical lobectomy [27], indicating that this membrane can be used in a practical manner in VATS in the clinical setting.

In the experimental group, only one animal (E4) developed an adhesion (left chest wall and lung) with an adhesion score of 3 (blunt dissection of the adhesion difficult to achieve), which can be clinically problematic with VATS. In this animal, the test membrane cracked during insertion and was torn into multiple pieces. The reason for the development of the clinically problematic adhesion was insufficient coverage of the target site. This animal did not show a pleural effusion at sacrifice. This was because the test membrane was manufactured by applying surface water induction technology, which enables integration of water by glycerol and then absorption of a large amount of water by insoluble hyaluronic acid to create a barrier [25]. Consequently, this physical property of this test membrane enables prevention of adhesions between the chest wall and lung. As possible causes of the development of adhesions, the test membrane may lose its physical properties as a barrier. By cracking the membrane during insertion, this membrane loses its surface water induction property, and the uncovered portion may develop adhesions.

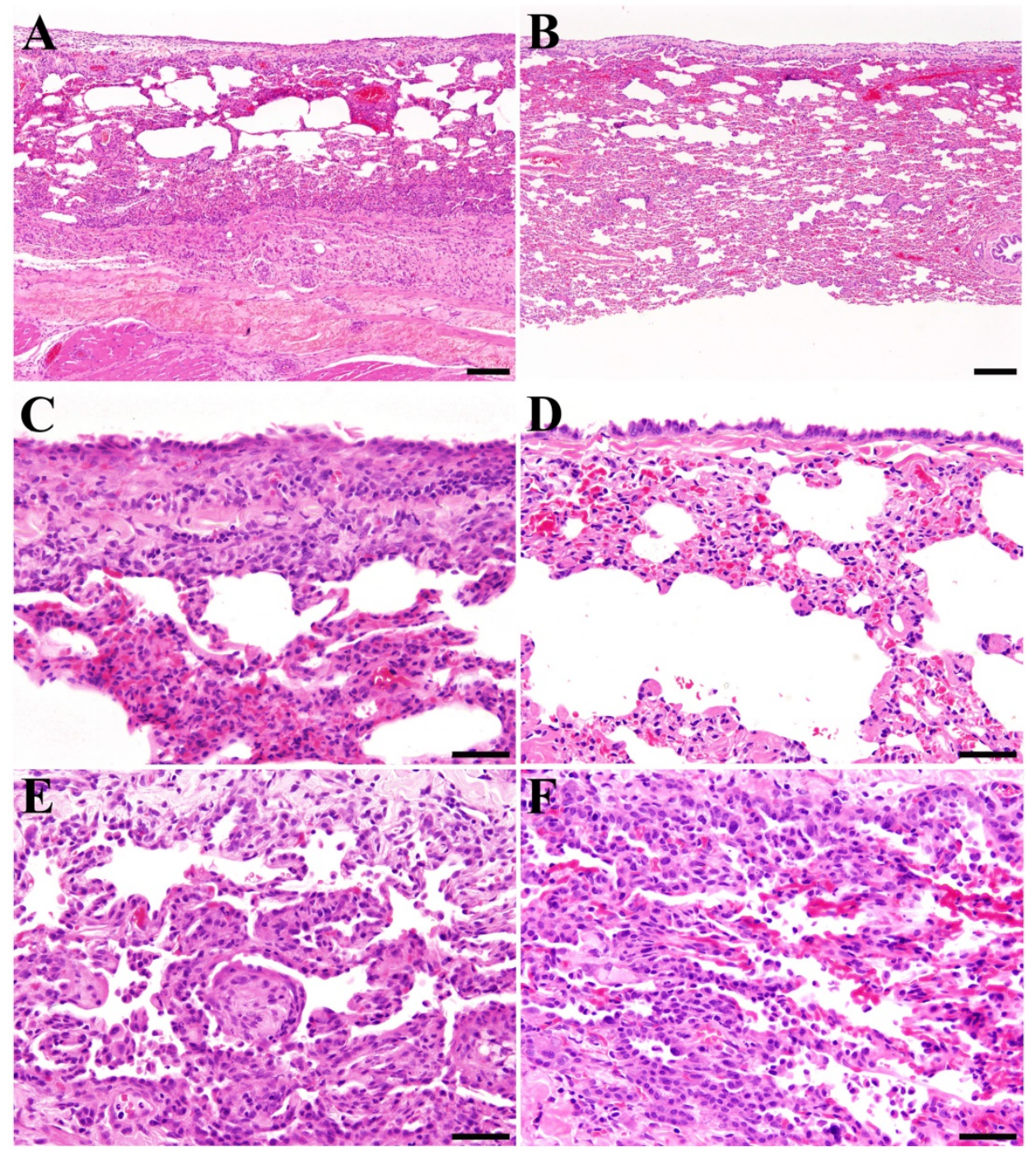

Fig 3. Histopathological changes in the chest wall and lungs at the re-thoracotomy. The test membrane elicits only a minor inflammatory response and foreign body reaction compared with the control group. (A, B) Pleural fibrosis (++) in a control animal (A) and an experimental group animal (B). (C, D) Mesothelial cell hypertrophy $(+)$ in a control animal (C) and an experimental group animal (D). (E, F) Alveolar epithelial cell cuboidal epithelialization (+) and mononuclear cell infiltration $(+)$ in a control animal $(E)$ and an experimental group animal $(F)$. (A-F: Hematoxylin and eosin staining. Bar: $A, B=200 \mu \mathrm{m}, x 10, C-F=50 \mu \mathrm{m}, \times 40$. 
In the macroscopic examination at sacrifice, no exudative changes suggestive of inflammatory responses were observed in the thoracic cavity in the experimental group. Histopathological analysis also showed no apparent induction of inflammatory changes or a foreign body reaction. This result suggests that the test membrane dissolves spontaneously within the thoracic cavity to be absorbed into the body and does not remain as a foreign substance, thus not causing an inflammatory response or foreign body reaction. Although the chest drain was removed a few days postoperatively when a pleural effusion was no longer observed, a pale yellow pleural effusion $(90-110 \mathrm{~mL} / \mathrm{dog})$ was observed in 4/5 animals in the experimental group at sacrifice. This pale yellow pleural effusion might be generated during the process to dissolve and absorb the test membrane. In human medicine, the clinical symptoms of pleural effusions are considered to be dependent on the underlying lung disease [23, 28, 29], and the pleural effusion observed in the present study would be unlikely to become a problem in the clinical setting.

The extent of surgical invasiveness is one of the important issues that must be addressed. While postoperative adhesions after thoracic surgery occurs at a high rate [5-7, 9], the incidence of adhesions depends on the intraoperative invasiveness of the surgical procedure. In the present study, only wedge resection of the lung lobe was performed using an automatic suture device. In this procedure, intraoperative tissue dissection was not necessary, and only a small amount of bleeding can be expected. This is the reason for the fewer adhesions that occurred even in the control group, and VATS is less invasive than thoracotomy. Actually, problems with postoperative adhesions have been described in VATS [10-12]. Therefore, in future investigations, induction of adhesions will need to be compared between use and non-use of the test membrane in surgeries with a greater degree of invasiveness, such as lobectomy or pericardial incision, instead of wedge resection.

In conclusion, the test membrane used in this study showed satisfactory operability not only in typical thoracotomy, but also in VATS, which is becoming increasingly common. Its easy delivery and spread within the thoracic cavity may be well suited for VATS procedures. This test membrane elicited only a minor inflammatory response and foreign body reaction and appears to be practical as a material to prevent postoperative adhesions after thoracic surgery and VATS.

\section{Acknowledgments}

The authors would like to thank Dainichiseika
Color \& Chemicals Mfg. Co., Ltd. for providing the test membrane samples used in this study.

\section{Author Contributions}

AU summarized the experimental findings, wrote the main manuscript text, and prepared Tables 1 and 2 and Figures 1 and 2. TF and TT were the anesthetists or assistants during the implantation of the experimental test membrane. $\mathrm{YH}$ and $\mathrm{MS}$ prepared Figure 3 and performed histopathological diagnoses. RT was the primary investigator and was responsible for the study design and funding. All authors reviewed the manuscript.

$R T$ received funding from the Japan Science and Technology Agency (National Research and Development Agency) as the Head of the Department of Veterinary Surgery of Tokyo University of Agriculture and Technology and these funds were used to purchase the dogs and medicine used in this study. AU, TF and TT used these dogs and medicine for this study. YH and MS declare no potential conflict of interest.

\section{Competing Interests}

This study was funded by the Japan Science and Technology Agency (National Research and Development Agency). The sponsor played no role in the collection, analysis or interpretation of data; in the writing of the report; or in the decision to submit the article for publication.

This research was carried out as a collaborative development project contracted by the Japan Science and Technology Agency as recommissioned research for a membrane using surface water induction technology to prevent pleural adhesions. This research was funded by grants from the Japan Science and Technology Agency (grant number: 5160545201).

\section{References}

1. Hong G, Vilz TO, Kalff JC, Wehner S. [Peritoneal adhesion formation]. Chirurg. 2015; 86: 175-80.

2. ten Broek RP, Issa Y, van Santbrink EJ, Bouvy ND, Kruitwagen RF, Jeekel J, et al. Burden of adhesions in abdominal and pelvic surgery: systematic review and met-analysis. BMJ. 2013; 347: f5588.

3. Muschalla F, Schwarz J, Bittner R. Effectivity of laparoscopic inguinal hernia repair (TAPP) in daily clinical practice: early and long-term result. Surg Endosc. 2016; 30: 4985-94

4. Satoh H, Kurishima K, Kagohashi K. Pneumothorax with postoperative complicated pleural adhesion. Tuberk Toraks. 2013; 61: 357-9.

5. Oizumi H, Naruke T, Watanabe H, Sano T, Kondo H, Goya T, et al. [Completion pneumonectomy--a review of 29 cases]. Nihon Kyobu Geka Gakkai Zasshi. 1990; 38: 72-7.

6. Yim AP, Liu HP, Hazelrigg SR, Izzat MB, Fung AL, Boley TM, et al. Thoracoscopic operations on reoperated chests. Ann Thorac Surg. 1998; 65: 328-30.

7. Getman V, Devyatko E, Wolner E, Aharinejad S, Mueller MR. Fleece bound sealing prevents pleural adhesions. Interact Cardiovasc Thorac Surg. 2006; 5: 243-6.

8. Loop FD. Catastrophic hemorrhage during sternal reentry. Ann Thorac Surg. 1984; 37: 271-2.

9. Braxton JH, Higgins RS, Schwann TA, Sanchez JA, Dewar ML, Kopf GS, et al. Reoperative mitral valve surgery via right thoracotomy: decreased blood loss and improved hemodynamics. J Heart Valve Dis. 1996; 5: 169-73. 
10. Buyukkale S, Citak N, Isgorucu O, Sayar A. A bioabsorbable membrane $($ Seprafilm(R)) may prevent postoperative mediastinal adhesions following mediastinoscopy: an experimental study in rats. Int J Clin Exp Med. 2015; 8: 11544-8.

11. Bruggmann D, Tchartchian G, Wallwiener M, Munstedt K, Tinneberg HR, Hackethal A. Intra-abdominal adhesions: definition, origin, significance in surgical practice, and treatment options. Dtsch Arztebl Int. 2010; 107: 769-75.

12. van Goor H. Consequences and complications of peritoneal adhesions. Colorectal Dis. 2007; 9 Suppl 2: 25-34.

13. Diamond MP, Burns EL, Accomando B, Mian S, Holmdahl L. Seprafilm((R)) adhesion barrier: (2) a review of the clinical literature on intraabdominal use. Gynecol Surg. 2012; 9: 247-57.

14. Ozerhan IH, Urkan M, Meral UM, Unlu A, Ersoz N, Demirag F, et al. Comparison of the effects of Mitomycin-C and sodium hyaluronate/carboxymethylcellulose [NH/CMC] (Seprafilm) on abdominal adhesions. Springerplus. 2016; 5: 846.

15. Lefort B, El Arid JM, Bouquiaux AL, Soule N, Chantreuil J, Tavernier E, et al. Is Seprafilm valuable in infant cardiac redo procedures? J Cardiothorac Surg. 2015; $10: 47$.

16. Sumi Y, Yamashita K, Kanemitsu K, Yamamoto M, Kanaji S, Imanishi T, et al. Simple and Easy Technique for the Placement of Seprafilm During Laparoscopic Surgery. Indian J Surg. 2015; 77: 1462-5.

17. Tsuruta A, Itoh T, Hirai T, Nakamura M. Multi-layered intra-abdominal adhesion prophylaxis following laparoscopic colorectal surgery. Surg Endosc. 2015; 29: 1400-5.

18. Koketsu S, Sameshima S, Okuyama T, Yamagata Y, Takeshita E, Tagaya N, et al. An effective new method for the placement of an anti-adhesion barrier film using an introducer in laparoscopic surgery. Tech Coloproctol. 2015; 19: 551-3.

19. Altuntas YE, Kement M, Oncel M, Sahip Y, Kaptanoglu L. The effectiveness of hyaluronan-carboxymethylcellulose membrane in different severity of adhesions observed at the time of relaparotomies: an experimental study on mice. Dis Colon Rectum. 2008; 51: 1562-5.

20. Kusuki I, Suganuma I, Ito F, Akiyama M, Sasaki A, Yamanaka K, et al. Usefulness of moistening seprafilm before use in laparoscopic surgery. Surg Laparosc Endosc Percutan Tech. 2014; 24: e13-5.

21. Takagi K, Tsuchiya T, Araki M, Yamasaki N, Nagayasu T, Hyon SH, et al. Novel biodegradable powder for preventing postoperative pleural adhesion. J Surg Res. 2013; 179: e13-9.

22. Izumi Y, Takahashi Y, Kohno M, Nomori H. Cross-linked poly(gamma-glutamic acid) attenuates pleural and chest wall adhesions in a mouse thoracotomy model. Eur Surg Res. 2012; 48: 93-8.

23. Karkhanis VS, Joshi JM. Pleural effusion: diagnosis, treatment, and management. Open Access Emerg Med. 2012; 4: 31-52.

24. Akerberg D, Posaric-Bauden M, Isaksson K, Andersson R, Tingstedt B. Prevention of pleural adhesions by bioactive polypeptides - a pilot study. Int J Med Sci. 2013; 10: 1720-6.

25. Noishiki Y, Shintani N. Anti-adhesive membrane for pleural cavity. Artif Organs. 2010; 34: 224-9.

26. Uemura A, Nakata M, Goya S, Fukayama T, Tanaka R. Effective new membrane for preventing postthoracotomy pleural adhesion by surface water induction technology. PLoS One. 2017; 12: e0179815.

27. Carrott PW, Jr., Jones DR. Teaching video-assisted thoracic surgery (VATS) lobectomy. J Thorac Dis. 2013; 5 Suppl 3: S207-11.

28. Yu H. Management of pleural effusion, empyema, and lung abscess. Semin Intervent Radiol. 2011; 28: 75-86.

29. Na MJ. Diagnostic tools of pleural effusion. Tuberc Respir Dis (Seoul). 2014; 76: 199-210. 